\title{
Aplicaciones de un ordenador de proceso IBM 1800, en una fábrica de cemento española (*)
}

\section{JUAN PUIG MONTRAVETA \\ Licenciado en Ciencias Químicas \\ Jefe de Laboratorio de CEMENTOS MOLINS, S. A.}

El primer ordenador IBM de proceso fue instalado en España por la firma Cementos Molins, S. A., en su factoría de San Vicente dels Horts (Barcelona), en julio de 1970.

Sus principales aplicaciones, presentes o futuras, se pueden agrupar en las siguientes actividades:

A) Actividades de control de proceso:

1) Control de calidad del material crudo.

2) Supervisión del proceso: vigilancia de alarmas.

3) Control de las distintas fases del proceso (trituración, molienda, calcinación, etc.).

4) Control de expediciones.

5) Control de cantera.

6) Realización de partes periódicos, de marcha y control.

B) Actividades de tipo administrativo:

1) Realización de la nómina.

2) Realización de la facturación.

3) Control del almacén.

Para la realización de dichos trabajos, el ordenador IBM 1800 dispone del siguiente equipo:

Unidad central, con memoria de $16 \mathrm{~K}$, ampliable.

Dispositivos de recepción de hasta 200 entradas analógicas, y para la entrada-salida de unas 100 señales digitales. Ambos dispositivos son ampliables, para permitir alcanzar una mayor o total automatización del proceso.

Unidad de almacenamiento de discos con 3 cabezas lectoras-grabadoras, de las cuales 2 son para ser utilizadas en control de proceso y 1 para trabajos administrativos.

(*) Comunicación presentada en la IX Reunión Anual del COMMON.-Madrid 29 agosto, 1 septiembre 1972. 
Una máquina de escribir para entradas y salidas de datos del proceso, y dos impresoras para salidas de resultados.

Impresora rápida para la realización de trabajos administrativos.

Una perforadora, una lectora-perforadora y una verificadora de fichas.

Consola con teclado funcional, teclado numérico, registrador múltiple de curvas y displaydigital (tipo Nixe). Permitirá al operario de la sala de control conocer el valor de las variables conectadas al ordenador, modificar parámetros de los sistemas de control y poner o quitar diferentes bucles de fases del proceso, bajo control del ordenador.

El sistema operativo utilizado en el ordenador IBM 1800, es el Time Sharing Executive System, T.S.X., que permite simultanear el trabajo de proceso con el administrativo, con preferencia del primero.

El equipo humano que trabaja con el ordenador está formado por 1 analista, 1 programador y 1 perforador.

A continuación, se hará una exposición detallada del control de calidad del material crudo, por estar basada en una concepción original de técnicos españoles de IBM y de Cementos Molins, S. A.

Para la fabricación del cemento portland, según el procedimiento de vía seca, se trata de obtener un crudo, de granulometría y composición química adecuadas y constantes, a partir de la extracción, trituración, molienda y homogeneización de las materias primas. Se utilizan como materias primas caliza, arcilla, arenisca y pirita. Con este material crudo se alimenta al horno rotatorio, con recuperador de calor de 2 etapas, y del cual se obtiene el clínker portland, que al molturarse a una finura adecuada con la oportuna adición de yeso, da el producto final o cemento portland.

La obtención de un crudo adecuado y uniforme es el primer y más decisivo paso para garantizar la calidad y regularidad en la fabricación del cemento portland. Por ello, la primera meta a alcanzar por Cementos Molins, S. A. con la ayuda del ordenador de proceso, fue la de regular y automatizar el control del crudo.

Hasta 1970 no se disponía para ello de más medio que la determinación química de la titulación en carbonato del material crudo. seguida de la corrección manual de las proporciones de las materias primas utilizadas. Dicha titulación es el único análisis químico tradicional y rápido, si bien muy incompleto, a que se somete aún el crudo en muchas fábricas de cemento. La duración del análisis es de unos 15 minutos.

El análisis químico completo de un crudo, por vía tradicional, requiere unas 8 horas, tiempo excesivamente prolongado para una corrección continuada del proceso de molienda del material crudo.

En 1970, junto con la puesta en marcha del ordenador, se instalaron también un equipo multicanal de análisis por fluorescencia de rayos $\mathrm{X}$ y un equipo semiautomático de preparación de muestras en forma de perlas, ambos suministrados por Philips.

Con el equipo últimamente citado, la muestra de crudo es inicialmente calcinada y convertida después en una perla de bórax. El calentamiento se realiza mediante un generador de alta frecuencia. La duración total del proceso de preparación de muestra es de unos 7-8 minutos. Se trata de un equipo único en España, y de los que muy pocos funcionan en todo el mundo. 
La muestra de crudo así preparada, con forma análoga a la de un vidrio de un reloj de pulsera, es fijada en un portamuestras adecuado e introducida en el espectrómetro multicanal de rayos $\mathrm{X}$ para su análisis. El tiempo de análisis es de unos 3 a 4 minutos. En la actualidad, se analizan simultáneamente $\mathrm{CaO}, \mathrm{Fe}_{2} \mathrm{O}_{3}, \mathrm{Al}_{2} \mathrm{O}_{3}, \mathrm{SiO}_{2}$ y $\mathrm{MgO}$, esperando analizar en un futuro además $\mathrm{K}_{2} \mathrm{O}$ y $\mathrm{SO}_{3}$.

Los resultados analíticos del espectrómetro se transmiten a través de una máquina de escribir y se comunican automáticamente al ordenador, en forma de cuentas o impulsos recibidos por los detectores de cada uno de los canales analizadores. Es el propio ordenador el que, aplicando las curvas de calibrado del equipo de rayos $\mathrm{X}$ para cada tipo determinado de material a analizar, transforma dichos impulsos en concentraciones reales de la muestra que se analizó, y que además calcula los módulos de saturación en cal, de sílice y de alúmina, que son muy empleados en términos cementeros.

También el ordenador está conectado a los contadores-totalizadores de las básculas dosificadoras de las materias primas, de forma tal que puede leerlos y corregirlos automáticamente.

El control de calidad del crudo procedente de los molinos de las líneas III y IV, actualmente en funcionamiento, a partir de las lecturas de las básculas dosificadoras de las materias primas y de los resultados de análisis del equipo de rayos $\mathrm{X}$, se realiza mediante las siguientes etapas:

\section{Información de la toma de muestra}

El operario que retira las muestras de crudo de los molinos (una muestra de cada hora de molienda, en molinos alternos), cierra un contacto informando al ordenador sobre la hora y molino del que se retira la muestra.

El ordenador, que toma lecturas instantáneas de todas las básculas cada 15 segundos y las promedia e integra cada dos minutos, al informársele que se ha retirado una muestra procede a totalizar la producción correspondiente a la muestra retirada y a calcular los distintos porcentajes de las materias primas consumidas.

\section{Recepción e interpretación de los resultados de análisis}

La muestra retirada del molino de crudo, transformada en perla de bórax, es analizada por el espectrómetro multicanal de rayos X. Se codifica la muestra de forma tal que quede definida la naturaleza de la misma, para poder aplicar la correspondiente curva de calibrado, y su procedencia, para conocer el tratamiento de sus resultados.

Los resultados del análisis, expresados como número de impulsos por canal o compuesto analizado, se transforman en concentraciones y en módulos del crudo analizado.

Conocido el análisis medio del crudo existente en los silos de homogeneización antes de la toma de muestra y reactualizado al momento presente mediante los resultados de producción y análisis de la muestra actual, debe el ordenador decidir sobre cuales son los más idóneos porcentajes de las materias primas disponibles con que deben alimentarse los molinos, para alcanzar los módulos de consigna en el menor tiempo posible.

Ello sería relativamente sencillo si se conociera el análisis químico correcto de las materias primas de que se dispone en cada momento. La arenisca y la ceniza de pirita, que constituyen un 2-3\% y un 0,5-1\% del crudo respectivamente, poseen una composición 
química muy constante, confirmada por frecuentes análisis que periódicamente se van realizando. En cambio la caliza y la mezcla arcilla-caliza, que son los otros dos materiales que constituyen prácticamente el $95 \%$ o más del crudo, poseen unas composiciones químicas que fluctúan considerablemente. Debido a la dificultad de realizar una correcta toma de muestra de dichos materiales, y a la imprecisión que supondría considerar sus composiciones como constantes, se ha decidido realizar una estimación estadística de los mismos.

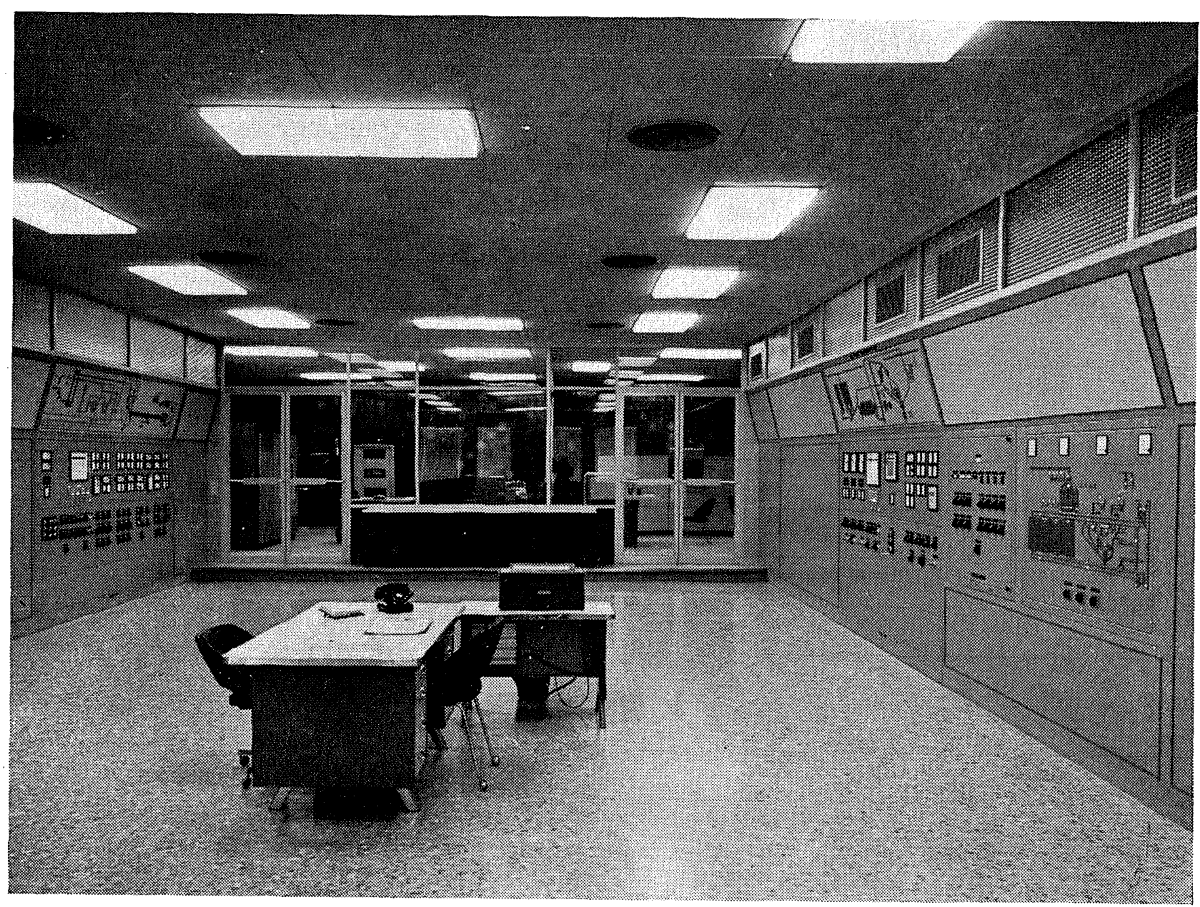

Foto 1.-Aspecto general de la sala central del control del proceso de fabricación.

\section{Estudio estadístico de la composición de materias primas}

A partir del análisis de la muestra de crudo, por medio del espectrómetro de rayos X. y de los porcentajes de las materias primas en la misma, se plantean las pertinentes ecuaciones que relacionan la concentración (en el crudo) de cada uno de los cumpuestos analizados $\left(\mathrm{CaO}, \mathrm{SiO}_{2}\right.$, etc.) en función de los porcentajes y contenidos, en cada compuesto, de las materias primas. En dichos sistemas de ecuaciones quedan como únicas incógnitas las que nos definen el análisis de las materias primas caliza y arcilla-caliza.

Al existir más incógnitas que ecuaciones, y para obtener un resultado estadístico suficientemente aproximado, habida cuenta de la variación con el tiempo de los análisis de dichas materias primas, se prepara una serie de ecuaciones igual a 10 . Se toman siempre la correspondiente a la última muestra analizada y a las 9 anteriores a ella. $\mathrm{Y}$ dicho sistema de ecuaciones se resuelve por el sistema de mínimos cuadrados.

A continuación se calcula el error o diferencia, en cada uno de los 5 compuestos analizados, entre el análisis real del crudo (por rayos $\mathrm{X}$ ) y el obtenido matemáticamente a partir de los porcentajes y análisis estadísticos o reales de las materias primas. Dichos porcentajes de error se distribuyen adecuadamente entre los análisis estadísticos de caliza y de arcilla-caliza. 
Todos los cálculos citados se realizan habitualmente cada media hora, reactualizándose pues, con dicha frecuencia, los análisis estadísticos de caliza y arcilla-caliza.

\section{Cálculo de nuevas dosificaciones de materias primas}

El material crudo deseado viene definido por unos determinados módulos de consigna, que a su vez corresponden a un cierto análisis químico.

A título meramente orientativo, se citan las definiciones de los módulos con que se opera:

Módulo o grado de saturación en cal: $\mathrm{LSF}=\frac{\mathrm{C}}{2,80 \mathrm{~S}+1,18 \mathrm{~A}+0,65 \mathrm{~F}}$

(Lime Saturation Factor).

Módulo de sílice

$$
\mathrm{M}_{\mathrm{S}}=\frac{\mathrm{S}}{\mathrm{A}+\mathrm{F}}
$$

Módulo de alúmina

$$
\mathrm{M}_{\mathrm{A}}=\frac{\mathrm{A}}{\mathrm{F}}
$$

Siendo: $\mathrm{C}=\mathrm{CaO} ; \mathrm{S}=\mathrm{SiO}_{2} ; \mathrm{A}=\mathrm{Al}_{2} \mathrm{O}_{3}$ y $\mathrm{F}=\mathrm{Fe}_{2} \mathrm{O}_{3}$.

Después de conocer el análisis y producción de la última muestra, el ordenador calcula el valor medio de los módulos del material existente en los silos, incluyendo el material que se ha molido en ambos molinos desde la última toma de muestra y que aún no se ha analizado. Para dicho material, su análisis se determina a partir de las composiciones reales o estadísticas de las materias primas.

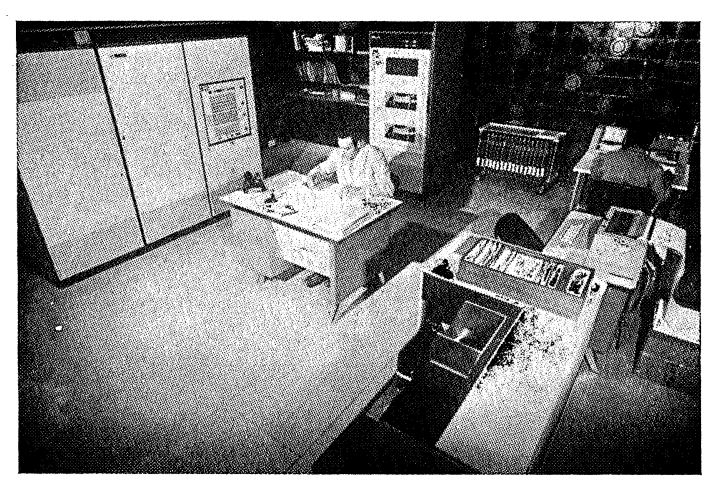

Foto 2

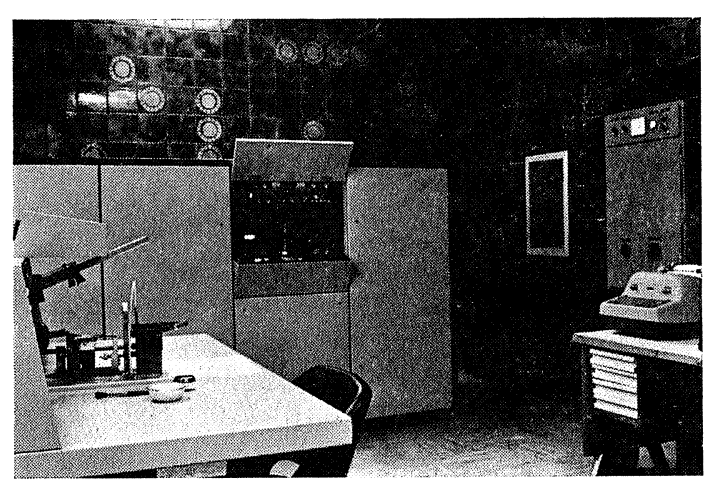

Foto 3

A partir de los módulos medios actuales del material crudo en los silos de homogeneización y de las composiciones químicas de las materias primas de que disponemos, el ordenador se plantea una serie de ecuaciones e inecuaciones, en programación lineal, y cuya solución permite determinar las proporciones óptimas de materias primas a utilizar en el próximo período, a fin de conseguir en el menor tiempo posible que los módulos medios coincidan. con ciertas tolerancias, con los módulos de consigna. 


\section{Corrección automática de las básculas dosificadoras}

A continuación, el ordenador manda una señal digital que conecta, durante un cierto período de tiempo, los motores reguladores de las básculas dosificadoras de las materias primas, a fin de conseguir que los porcentajes de las materias primas que alimentan al molino sean los que se calcularon con anterioridad (punto 4).

Al realizar el ordenador las lecturas de producciones y proporciones de materias primas en la molienda de crudo, cada dos minutos, si éstas no coinciden con los porcentajes calculados, el propio ordenador vuelve a modificar los potenciómetros de las básculas dosificadoras hasta alcanzar de nuevo los valores de consigna.

\section{Confección del parte de situación del control de crudo}

Una vez realizados todos los pasos anteriores, mediante una impresora de servicio informa al operario de la sala de control centralizado de la procedencia de la muestra analizada, la hora de toma de muestra, las toneladas a que corresponde ésta, los módulos de la misma, las proporciones de las distintas materias primas con que se alimentó el molino, los módulos medios del material en el silo, la cantidad total de material producido, y las nuevas proporciones de alimentación junto con la hora a que se realizó la corrección. Todos los datos citados aparecen en una sola línea, por lo cual es fácil de interpretar los resultados en dicho parte.

Un ciclo de control de calidad se considera concluido cuando el material crudo en los silos de homogeneización posee unos módulos medios coincidentes con los de consigna, o que están dentro de un estrecho margen de tolerancia con ellos. Entonces se habla de cerrar un ciclo de control e iniciar el siguiente. Normalmente dichos cierres se consiguen cada 3-4 horas, equivalente a una producción de 400-600 t de crudo.

Dentro del campo de aplicación del ordenador en el control del proceso, como primera etapa se le han conectado una serie de variables, como temperaturas, presiones, etc. $\mathrm{Pa}$ ra cada variable se ha realizado la correspondiente curva de calibrado y se le han asignado unos límites de funcionamiento normal. Periódicamente el ordenador lee todas las variables conectadas, cada 15 segundos y las promedia cada 2 minutos, y si cualquiera de ellas cae fuera de los límites habituales, emite un parte de alarma. En dicho parte notifica qué variable está fuera de límites, indicando si sobrepasó el límite superior o el inferior, así como si el valor cae fuera de los límites de lectura del instrumento medidor.

Como complemento de esta aplicación de supervisión, pueden almacenarse en la memoria de discos del ordenador los valores correspondientes a la evolución en el tiempo de ciertas variables, a partir de las lecturas medias de cada dos minutos, previamente filtradas. Estos datos pueden ser sometidos a estudios estadísticos, etc., a fin de elaborar un modelo matemático del proceso que permita en un futuro el establecimiento de sistemas de control.

En una primera fase, y en los molinos de crudo, el ordenador ha simulado a los reguladores P.I.D. que ya existían en los cuadros de control del proceso. Su funcionamiento es altamente satisfactorio. 